\title{
Solvability for generalized nonlinear two dimensional functional integral equations via measure of noncompactness
}

\author{
Soniya Singh', Bhupander Singh², Kottakkaran Sooppy Nisar ${ }^{3^{*}}$ (D), Abd-Allah Hyder ${ }^{4,5}$ and M. Zakarya $a^{4,6}$
}

\section{"Correspondence:}

n.sooppy@psau.edu.sa

${ }^{3}$ Department of Mathematics, College of Arts and Sciences, Prince Sattam bin Abdulaziz University, Wadi Aldawaser, Saudi Arabia Full list of author information is available at the end of the article

\begin{abstract}
In this article, we provide the existence result for functional integral equations by using Petryshyn's fixed point theorem connecting the measure of noncompactness in a Banach space. The results enlarge the corresponding results of several authors. We present fascinating examples of equations.
\end{abstract}

MSC: $45 \mathrm{A05} ; 45 \mathrm{H} 05$

Keywords: Petryshyn's fixed point theorem; Measure of noncompactness (in short $\mathrm{MNC}$ ); Functional integral equation (in short FIE)

\section{Introduction}

FIEs play a very significant role in many areas of fixed point theory, and they have many applications in various areas of mathematical physics, engineering, mathematical biology, population dynamics, natural science, and mechanics (see $[1,7,15,19,20,26,33])$. It has been seen that integral equations have a large number of applications to finding the existence solution of integro-differential equations, differential equations, and fractional differential equations. Recently, many authors have used the MNC technique associated with Darbo's fixed point theorem [3] to examine the existence and uniqueness results of various types of FIEs. The details of this type of work can be found in these articles (see $[4-6,8,9,11-14,17,18,24,25,30,32,34,35]$ and the references therein).

In this work, we use Petryshyn's fixed point theorem [29] instead of Darbo's fixed point theorem to establish the existence of solutions for the following FIE:

$$
\begin{aligned}
z(s, \zeta)= & G(s, \zeta)+F\left(s, \zeta, f(s, \zeta, z(s, \zeta)), \int_{0}^{s} \int_{0}^{\zeta} g(s, \zeta, \xi, \eta, z(\xi, \eta)) d \eta d \xi,\right. \\
& \left.\int_{0}^{c} \int_{0}^{d} h(s, \zeta, \xi, \eta, z(\xi, \eta)) d \eta d \xi\right),
\end{aligned}
$$

where $(s, \zeta) \in I=[0, c] \times[0, d]$. Recently several authors used Petryshyn's fixed point theorem to find the existence of solutions for nonlinear FIEs in Banach spaces as well as Banach

(c) The Author(s) 2021. This article is licensed under a Creative Commons Attribution 4.0 International License, which permits use, sharing, adaptation, distribution and reproduction in any medium or format, as long as you give appropriate credit to the original author(s) and the source, provide a link to the Creative Commons licence, and indicate if changes were made. The images or other third party material in this article are included in the article's Creative Commons licence, unless indicated otherwise in a credit line to the material. If material is not included in the article's Creative Commons licence and your intended use is not permitted by statutory regulation or exceeds the permitted use, you will need to obtain permission directly from the copyright holder. To view a copy of this licence, visit http://creativecommons.org/licenses/by/4.0/. 
algebra (for instance see $[10,21,22,31]$ and the references therein). The following statements explain the main causes why we use equation (1) and what is the perfection of our work. The first is that the conditions in various papers will be analyzed, and the second reason is that this paper unifies the relevant work in this area. The third condition is the bounded condition shows that the "sublinear condition" that has been discussed in several literature works does not have a significant role.

The paper is divided into five sections including the introduction. In Sect. 2, we present some preliminaries and define the concept of MNC. Section 3 states and proves an existence result for equations including condensing operators using Petryshyn's fixed point theorem. In Sect. 4 we give examples that test the utilization of this kind of FIE. Finally, Sect. 5 concludes the paper.

\section{Preliminaries}

In this work, $X$ is a real Banach space and $B_{\tilde{r}}$ denotes closed ball center at 0 with radius $\tilde{r}$ and $\partial B_{r}=\{z \in X:\|z\|=\tilde{r}\}$ for the sphere in $X$ around 0 with radius $\tilde{r}>0$. MNCs are valuable tools in the analysis of existence in the operator equations and theory of fixed point in $X$.

Definition 2.1 ([23]) Let $Y \in M_{X}$ and

$$
\mu(Y)=\inf \left\{\epsilon>0: Y=\bigcup_{i=1}^{n} Y_{i} \text { with } \operatorname{diam} Y_{i} \leq \epsilon, i=1,2, \ldots, n\right\} .
$$

Hence, $0 \leq \vartheta(Y)<\infty . \vartheta(Y)$ is called the Kuratowski MNC.

Definition 2.2 ([16]) The Hausdorff MNC

$$
\vartheta(Y)=\inf \{\epsilon>0 \text { : there exists a finite } \epsilon \text {-net for } Y \text { in } X\}
$$

where from a finite $\epsilon$-net for $Y$ in $X$ that means a set $\left\{z_{1}, z_{2}, \ldots, z_{n}\right\} \subset X$ such that the ball $B_{\epsilon}\left(X, z_{1}\right), B_{\epsilon}\left(X, z_{2}\right), \ldots, B_{\epsilon}\left(X, z_{n}\right)$ over $Y$. These MNCs are mutually equivalent in the sense that

$$
\vartheta(Y) \leq \hat{\beta}(Y) \leq 2 \vartheta(Y)
$$

for a bounded set $Y \subset X$.

Theorem 2.1 Let $Y, \hat{Y} \in M_{X}$ and $\lambda \in \mathbb{R}$. Then

(i) $\vartheta(Y)=0$ if and only if $Y \in M_{X}$;

(ii) $Y \subseteq \hat{Y}$ implies $\vartheta(Y) \leq \vartheta(\hat{Y})$;

(iii) $\vartheta(\operatorname{Conv} Y)=\vartheta(Y)$;

(iv) $\vartheta(Y \cup \hat{Y})=\max \{\vartheta(Y), \vartheta(\hat{Y})\}$;

(v) $\vartheta(\lambda Y)=|\lambda| \vartheta(Y)$

(vi) $\vartheta(Y+\hat{Y}) \leq \vartheta(Y)+\vartheta(\hat{Y})$.

Here, we consider the Banach space $C(I, \mathbb{R})$ with the usual norm

$$
\|z\|=\max \{|z(s, \zeta)|:(s, \zeta) \in I\} .
$$


Let $X \in C(I, \mathbb{R})$. Given $\epsilon>0$, the modulus of continuity of $z \in Y$ is defined as

$$
\omega(z, \epsilon)=\sup \{|z(s, \zeta)-z(\hat{s}, \hat{\zeta})|: s, \hat{s} \in[0, c], \zeta, \hat{\zeta} \in[0, d],|s-\hat{s}| \leq \epsilon,|\zeta-\hat{\zeta}| \leq \epsilon\}
$$

Further

$$
\omega(Y, \epsilon)=\sup \{\omega(z, \epsilon): z \in Y\}, \quad \omega_{0}(Y)=\lim _{\epsilon \rightarrow 0} \omega(Y, \epsilon) .
$$

Theorem 2.2 ([21]) The Hausdorff MNC is similar to

$$
\mu(Y)=\lim _{\epsilon \rightarrow 0} \sup \omega(z, \epsilon)
$$

for all bounded set $Y \subset C(I, \mathbb{R})$.

Theorem 2.3 ([27]) Let $H: X \rightarrow X$ be a continuous mapping of $X . H$ is called a $k$ set contraction if, for all $D \subset X$ with $D$ bounded, $H(D)$ is bounded and $\hat{\beta}(H D) \leq k \hat{\beta}(D), k \in$ $(0,1)$. If $\hat{\beta}(H D)<\hat{\beta}(D)$ for all $\hat{\beta}(D)>0$, then $H$ is called densifying or condensing map.

Theorem 2.4 ([29]) Let $H: B_{\tilde{r}} \rightarrow X$ be a condensing function which fulfills the boundary condition if $H(z)=k z$ for some $z \in \partial B_{r}$, then $k \leq 1$. Then $F(H)$ in $B_{\tilde{r}}$ is nonempty, where $F(H)$ is the set of fixed points of $H$.

\section{Main results}

Now, we study the main aim of equation (1). Namely, we assume the following assumptions:

(1) $G \in C(I, \mathbb{R}), F \in C\left(I_{1} \times \mathbb{R} \times \mathbb{R}, \mathbb{R}\right), f \in C(I \times \mathbb{R}, \mathbb{R}), g, h \in C\left(I_{2} \times \mathbb{R}, \mathbb{R}\right)$, where

$$
\begin{aligned}
& I=I_{c} \times I_{d}, \quad I_{1}=\{(s, \zeta, f): 0 \leq s \leq c, 0 \leq \zeta \leq d, \xi \in \mathbb{R}\}, \\
& I_{2}=\left\{(s, t, \xi, \eta) \in I^{2}: 0 \leq \xi \leq s \leq c, 0 \leq \eta \leq \zeta \leq d\right\}
\end{aligned}
$$

(2) There exist nonnegative constants $k_{1}, k_{2}, k_{3}, k_{4}, k_{1} k_{4}<1$ such that

$$
\begin{aligned}
& \mid F(s, \zeta, z, u, x)-F\left(s, \zeta, \hat{z}, \hat{u}, \hat{x}\left|\leq k_{1}\right| z-\hat{z}\left|+k_{2}\right| u-\hat{u}\left|+k_{3}\right| x-\hat{x} \mid ;\right. \\
& \mid f(s, \zeta, z)-f\left(s, \zeta, \hat{z}\left|\leq k_{4}\right| z-\hat{z} \mid ;\right.
\end{aligned}
$$

(3) There exists $\tilde{r}>0$ such that the resulting bounded condition is fulfilled

$$
\begin{gathered}
\sup \{|G(s, \zeta):(s, \zeta) \in I|+|F(s, \zeta, z, u, x)|:(s, \zeta) \in I, z \in[-\tilde{r}, \tilde{r}], \\
\left.u \in\left[-c d M_{1}, c d M_{1}\right], x \in\left[-c d M_{2}, c d M_{2}\right]\right\} \leq \tilde{r}
\end{gathered}
$$

where

$$
\begin{aligned}
& M_{1}=\sup \left\{|g(s, \zeta, \xi, \eta, z)|: \text { for all }(s, \zeta, \xi, \eta) \in I_{2} \text { and } z \in[-\tilde{r}, \tilde{r}]\right\} \\
& M_{2}=\sup \left\{|h(s, \zeta, \xi, \eta, z)|: \text { for all }(s, \zeta, \xi, \eta) \in I_{2} \text { and } z \in[-\tilde{r}, \tilde{r}]\right\}
\end{aligned}
$$


Theorem 3.1 Under assumptions (1)-(3) with $k_{1} k_{4}<1$, equation (1) has at least one solution in $X$.

Proof Define $H: B_{\tilde{r}} \rightarrow X$ in the following form:

$$
\begin{aligned}
(H z)(s, \zeta)= & G(s, \zeta)+F\left(s, \zeta, f(s, \zeta, z(s, \zeta)), \int_{0}^{s} \int_{0}^{\zeta} g(s, \zeta, \xi, \eta, z(\xi, \eta)) d \eta d \xi,\right. \\
& \left.\int_{0}^{c} \int_{0}^{d} h(s, \zeta, \xi, \eta, z(\xi, \eta)) d \eta d \xi\right) .
\end{aligned}
$$

Now, we show that $H$ is continuous on the ball $B_{\tilde{r}}$. Take $\epsilon>0$ and $z, x \in B_{\tilde{r}}$ such that $\| z-$ $x \|<\epsilon$. We get

$$
\begin{aligned}
|(H z)(s, \zeta)-(H x)(s, \zeta)| & \mid G(s, \zeta)+F\left(s, \zeta, f(s, \zeta, z(s, \zeta)), \int_{0}^{s} \int_{0}^{\zeta} g(s, \zeta, \xi, \eta, z(\xi, \eta)) d \eta d \xi,\right. \\
& \left.\int_{0}^{c} \int_{0}^{d} h(s, \zeta, \xi, \eta, z(\xi, \eta)) d \eta d \xi\right) \\
& -G(s, \zeta)-F\left(s, \zeta, f(s, \zeta, x(s, \zeta)), \int_{0}^{s} \int_{0}^{\zeta} g(s, \zeta, \xi, \eta, x(\xi, \eta)) d \eta d \xi\right. \\
& \left.\int_{0}^{c} \int_{0}^{d} h(s, \zeta, \xi, \eta, x(\xi, \eta)) d \eta d \xi\right) \mid \\
\leq & k_{1}|f(s, \zeta, z(s, \zeta))-f(s, \zeta, x(s, \zeta))| \\
& +k_{2} \int_{0}^{s} \int_{0}^{\zeta}|g(s, \zeta, \xi, \eta, z(\xi, \eta))-g(s, \zeta, \xi, \eta, x(\xi, \eta))| d \eta d \xi \\
& +k_{3} \int_{0}^{c} \int_{0}^{d}|h(s, \zeta, \xi, \eta, z(\xi, \eta))-h(s, \zeta, \xi, \eta, x(\xi, \eta))| d \eta d \xi \\
\leq & k_{1} k_{4}|z(s, \zeta)-x(s, \zeta)|+k_{2} c d \omega(g, \epsilon)+k_{3} c d \omega(h, \epsilon) \\
\leq & k_{1} k_{4}\|z-x\|+k_{2} c d \omega(g, \epsilon)+k_{3} c d \omega(h, \epsilon),
\end{aligned}
$$

where, for $\epsilon>0$, we denote

$$
\begin{aligned}
& \omega(g, \epsilon)=\sup \left\{|g(s, \zeta, \xi, \eta, z)-g(s, \zeta, \xi, \eta, x)|:(s, \zeta, \xi, \eta) \in I_{2}, z, x \in[-\tilde{r}, \tilde{r}],\|z-x\| \leq \epsilon\right\} \\
& \omega(h, \epsilon)=\sup \left\{|h(s, \zeta, \xi, \eta, z)-h(s, \zeta, \xi, \eta, x)|:(s, \zeta, \xi, \eta) \in I_{2}, z, x \in[-\tilde{r}, \tilde{r}],\|z-x\| \leq \epsilon\right\} .
\end{aligned}
$$

Now, from the uniform continuity of $g(s, \zeta, \xi, \eta, z)$ and $h(s, \zeta, \xi, \eta, z)$ on $I_{2} \times[-\epsilon, \epsilon]$ respectively, then $\omega(g, \epsilon)$ and $\omega(h, \epsilon)$ as $\epsilon \rightarrow 0$. Hence, we decide that $H$ is continuous on $B_{\tilde{r}}$.

Next, we prove that $H$ fulfills the densifying condition. Select $\epsilon>0$ and take $z \in Y$, where $Y$ is a bounded subset of $X,\left(s_{1}, \zeta_{1}\right),\left(s_{2}, \zeta_{2}\right) \in I$ with $s_{1} \leq s_{2}, \zeta_{1} \leq \zeta_{2}$ such that $s_{1}-s_{2} \leq \epsilon$, $\zeta_{1}-\zeta_{2} \leq \epsilon$, we obtain

$$
\begin{aligned}
& \left|(H z)\left(s_{2}, \zeta_{2}\right)-(H z)\left(s_{1}, \zeta_{1}\right)\right| \\
& \quad=\mid G\left(s_{2}, \zeta_{2}\right)+F\left(s_{2}, \zeta_{2}, f\left(s_{2}, \zeta_{2}, z\left(s_{2}, \zeta_{2}\right)\right), \int_{0}^{s_{2}} \int_{0}^{\zeta_{2}} g\left(s_{2}, \zeta_{2}, \xi, \eta, z(\xi, \eta)\right) d \eta d \xi\right.
\end{aligned}
$$




$$
\begin{aligned}
& \left.\int_{0}^{c} \int_{0}^{d} h\left(s_{2}, \zeta_{2}, \xi, \eta, z(\xi, \eta)\right) d \eta d \xi\right) \\
& -G\left(s_{1}, \zeta_{1}\right)-F\left(s_{1}, \zeta_{1}, f\left(s_{1}, \zeta_{1}, z\left(s_{1}, \zeta_{1}\right)\right), \int_{0}^{s_{1}} \int_{0}^{\zeta_{1}} g\left(s_{1}, \zeta_{1}, \xi, \eta, z(\xi, \eta)\right) d \eta d \xi,\right. \\
& \left.\int_{0}^{c} \int_{0}^{d} h\left(s_{1}, \zeta_{1}, \xi, \eta, z(\xi, \eta)\right) d \eta d \xi\right) \mid \\
& \leq \omega_{1}(G, \epsilon)+\mid F\left(s_{2}, \zeta_{2}, f\left(s_{2}, \zeta_{2}, z\left(s_{2}, \zeta_{2}\right)\right), \int_{0}^{s_{2}} \int_{0}^{\zeta_{2}} g\left(s_{2}, \zeta_{2}, \xi, \eta, z(\xi, \eta)\right) d \eta d \xi,\right. \\
& \left.\int_{0}^{c} \int_{0}^{d} h\left(s_{2}, \zeta_{2}, \xi, \eta, z(\xi, \eta)\right) d \eta d \xi\right) \\
& -F\left(s_{2}, \zeta_{2}, f\left(s_{2}, \zeta_{2}, z\left(s_{2}, \zeta_{2}\right)\right), \int_{0}^{s_{2}} \int_{0}^{\zeta_{2}} g\left(s_{2}, \zeta_{2}, u, \xi, z(\xi, \eta)\right) d \eta d \xi,\right. \\
& \left.\int_{0}^{c} \int_{0}^{d} h\left(s_{1}, \zeta_{1}, \xi, \eta, z(\xi, \eta)\right) d \eta d \xi\right) \\
& +\mid F\left(s_{2}, \zeta_{2}, f\left(s_{2}, \zeta_{2}, z\left(s_{2}, \zeta_{2}\right)\right), \int_{0}^{s_{2}} \int_{0}^{\zeta_{2}} g\left(s_{2}, \zeta_{2}, u, \xi, z(\xi, \eta)\right) d \eta d \xi,\right. \\
& \left.\left.\int_{0}^{c} \int_{0}^{d} h\left(s_{1}, \zeta_{1}, \xi, \eta, z(\xi, \eta)\right) d \eta d \xi\right) \mid\right) \\
& -F\left(s_{2}, \zeta_{2}, f\left(s_{2}, \zeta_{2}, z\left(s_{2}, \zeta_{2}\right)\right), \int_{0}^{s_{1}} \int_{0}^{\zeta_{1}} g\left(s_{1}, \zeta_{1}, u, \xi, z(\xi, \eta)\right) d \eta d \xi,\right. \\
& \int_{0}^{c} \int_{0}^{d} h\left(s_{1}, \zeta_{1}, \xi, \eta, z(\xi, \eta)\right) d \eta d \xi \\
& +\mid F\left(s_{2}, \zeta_{2}, f\left(s_{2}, \zeta_{2}, z\left(s_{2}, \zeta_{2}\right)\right), \int_{0}^{s_{1}} \int_{0}^{\zeta_{1}} g\left(s_{1}, \zeta_{1}, u, \xi, z(\xi, \eta)\right) d \eta d \xi,\right. \\
& \left.\int_{0}^{c} \int_{0}^{d} h\left(s_{1}, \zeta_{1}, \xi, \eta, z(\xi, \eta)\right) d \eta d \xi\right) \\
& -F\left(s_{2}, \zeta_{2}, f\left(s_{1}, \zeta_{1}, z\left(s_{1}, \zeta_{1}\right)\right), \int_{0}^{s_{1}} \int_{0}^{\zeta_{1}} g\left(s_{1}, \zeta_{1}, u, \xi, z(\xi, \eta)\right) d \eta d \xi,\right. \\
& \left.\int_{0}^{c} \int_{0}^{d} h\left(s_{1}, \zeta_{1}, \xi, \eta, z(\xi, \eta)\right) d \eta d \xi\right) \\
& +\mid F\left(s_{2}, \zeta_{2}, f\left(s_{1}, \zeta_{1}, z\left(s_{1}, \zeta_{1}\right)\right), \int_{0}^{s_{1}} \int_{0}^{\zeta_{1}} g\left(s_{1}, \zeta_{1}, u, \xi, z(\xi, \eta)\right) d \eta d \xi,\right. \\
& \left.\int_{0}^{c} \int_{0}^{d} h\left(s_{1}, \zeta_{1}, \xi, \eta, z(\xi, \eta)\right) d \eta d \xi\right) \\
& -F\left(s_{1}, \zeta_{1}, f\left(s_{1}, \zeta_{1}, z\left(s_{1}, \zeta_{1}\right)\right), \int_{0}^{s_{1}} \int_{0}^{\zeta_{1}} g\left(s_{1}, \zeta_{1}, u, \xi, z(\xi, \eta)\right) d \eta d \xi,\right. \\
& \left.\int_{0}^{c} \int_{0}^{d} h\left(s_{1}, \zeta_{1}, \xi, \eta, z(\xi, \eta)\right) d \eta d \xi\right) \\
& \leq k_{3}\left|\int_{0}^{c} \int_{0}^{d} h\left(s_{2}, \zeta_{2}, u, \xi, z(u, \xi)\right) d \eta d \xi-\int_{0}^{c} \int_{0}^{d} h\left(s_{1}, \zeta_{1}, u, \xi, z(u, \xi)\right) d \eta d \xi\right| \\
& +k_{2}\left|\int_{0}^{s_{2}} \int_{0}^{\zeta_{2}} g\left(s_{2}, \zeta_{2}, \xi, \eta, z(\xi, \eta)\right) d \eta d \xi-\int_{0}^{s_{1}} \int_{0}^{t_{1}} g\left(s_{1}, \zeta_{1}, \xi, \eta, z(\xi, \eta)\right) d \eta d \xi\right|
\end{aligned}
$$




$$
\begin{aligned}
& +k_{1}\left|f\left(s_{2}, \zeta_{2}, z\left(s_{2}, \zeta_{2}\right)\right)-f\left(s_{2}, \zeta_{2}, z\left(s_{1}, \eta_{1}\right)\right)\right|+k_{1} \mid f\left(s_{2}, \zeta_{2}, z\left(s_{1}, \zeta_{1}\right)\right) \\
& -s\left(s_{1}, \zeta_{1}, z\left(s_{1}, \zeta_{1}\right)\right) \mid+\omega_{1}(G, \epsilon)+\omega_{1}(F, \epsilon) \\
\leq & k_{3} \int_{0}^{c} \int_{0}^{d}\left|h\left(s_{2}, \zeta_{2}, \xi, \eta, z(\xi, \eta)\right)-h\left(s_{1}, \zeta_{1}, \xi, \eta, z(\xi, \eta)\right)\right| d \eta d \xi \\
& +k_{2} \int_{0}^{s_{1}} \int_{0}^{\zeta_{1}}\left|g\left(s_{2}, \zeta_{2}, \xi, \eta, z(\xi, \eta)\right)-g\left(s_{1}, \zeta_{1}, \xi, \eta, z(\xi, \eta)\right)\right| d \eta d \xi \\
& +k_{2} \int_{s_{1}}^{s_{2}} \int_{0}^{\zeta_{1}}\left|g\left(s_{2}, \zeta_{2}, \xi, \eta, z(\xi, \eta)\right)\right| d \eta d \xi+\omega_{1}(G, \epsilon)+\omega_{1}(F, \epsilon) \\
& +k_{2} \int_{0}^{s_{1}} \int_{\zeta_{1}}^{\zeta_{2}}\left|g\left(s_{2}, \zeta_{2}, \xi, \eta, z(\xi, \eta)\right)\right| d \eta d \xi+k_{1} k_{4}\left|z\left(s_{2}, \zeta_{2}\right)-z\left(s_{1}, \zeta_{1}\right)\right| \\
& +k_{2} \int_{s_{1}}^{s_{2}} \int_{\zeta_{1}}^{\zeta_{2}}\left|g\left(s_{2}, \zeta_{2}, \xi, \eta, z(\xi, \eta)\right)\right| d \eta d \xi+k_{1} \omega_{1}(f, \epsilon),
\end{aligned}
$$

where

$$
\begin{aligned}
\omega_{1}(f, \epsilon)= & \sup \{|f(s, \zeta, z)-f(\hat{s}, \hat{\zeta}, z)|:|s-\hat{s}| \leq \epsilon,|\zeta-\hat{\zeta}| \leq \epsilon, z \in[-\tilde{r}, \tilde{r}]\}, \\
\omega_{1}(g, \epsilon)= & \sup \{|g(s, \zeta, \xi, \eta, z)-g(\hat{s}, \hat{\zeta}, \xi, \eta, z)|:|s-\hat{s}| \leq \epsilon,|\zeta-\hat{\zeta}| \leq \epsilon, \\
& \left.(s, \zeta, \xi, \eta) \in I_{2}, z \in[-\tilde{r}, \tilde{r}]\right\}, \\
\omega_{1}(h, \epsilon)= & \sup \{|h(s, \zeta, \xi, \eta, z)-h(\hat{s}, \hat{\zeta}, \xi, \eta, z)|:|s-\hat{s}| \leq \epsilon,|\zeta-\hat{\zeta}| \leq \epsilon, \\
& \left.(s, \zeta, \xi, \eta) \in I_{2}, z \in[-\tilde{r}, \tilde{r}]\right\} \\
\omega_{1}(F, \epsilon)= & \sup \left\{|F(s, \zeta, z, u, x)-s(\hat{s}, \hat{\zeta}, z, u, x)|:|s-\hat{s}| \leq \epsilon,|\zeta-\hat{\zeta}| \leq \epsilon, z_{1} \in[-\tilde{r}, \tilde{r}],\right. \\
& \left.u \in\left[-c d M_{1}, c d M_{1}\right], x \in\left[-c d M_{2}, c d M_{2}\right]\right\} .
\end{aligned}
$$

Then, using the above relation, we get

$$
\begin{aligned}
& \left|(H z)\left(s_{2}, \zeta_{2}\right)-(H z)\left(s_{1}, \zeta_{1}\right)\right| \\
& \quad \leq k_{1} k_{4}\left|z\left(s_{2}, v_{2}\right)-z\left(s_{1}, \zeta_{1}\right)\right|+k_{1} \omega_{1}(f, \epsilon)+\omega_{1}(F, \epsilon) \\
& \quad+k_{3} c d \omega_{1}(h, \epsilon)+k_{2} c d \omega(g, \epsilon)+\epsilon k_{2} d M_{1}+\epsilon k_{2} c M_{1}+\epsilon^{2} k_{2} M_{1} .
\end{aligned}
$$

Applying limit as $\delta \rightarrow 0$,

$$
\omega(H z, \epsilon) \leq k_{1} k_{4} \omega(z, \epsilon)
$$

This gives the following relation:

$$
\vartheta(H Y) \leq k_{1} k_{4} \vartheta(Y)
$$

hence $H$ is a condensing map. Now, let $z \in \partial B_{\tilde{r}}$, and if $H z=k z$, then $\|H z\|=k\|z\|=k \tilde{r}$, and by (3), we obtain

$$
|H z(s, \zeta)|=G(s, \zeta)+F\left(s, \zeta, f(s, \zeta, z(s, \zeta)), \int_{0}^{s} \int_{0}^{\zeta} g(s, \zeta, \xi, \eta, z(\xi, \eta)) d \eta d \xi,\right.
$$




$$
\left.\int_{0}^{c} \int_{0}^{d} h(s, \zeta, \xi, \eta, z(\xi, \eta)) d \eta d \xi\right)
$$

for all $(s, \zeta) \in I$. Hence $\|H z\| \leq \tilde{r}$ i.e. $k \leq 1$.

Corollary 3.2 Let

(1) $G \in C(I, \mathbb{R}), F \in C\left(I_{1} \times \mathbb{R} \times \mathbb{R}, \mathbb{R}\right), g, h \in C\left(I_{2} \times \mathbb{R}, \mathbb{R}\right)$, where

$$
\begin{aligned}
& I=I_{c} \times I_{d}, \quad I_{1}=\{(s, \zeta, z): 0 \leq s \leq c, 0 \leq \zeta \leq d, s \in \mathbb{R}\} \\
& I_{2}=\left\{(s, \zeta, \xi, \eta) \in I^{2}: 0 \leq \xi \leq s \leq c, 0 \leq \eta \leq \zeta \leq d\right\}
\end{aligned}
$$

(2) There exist nonnegative constants $k_{1}, k_{2}, k_{3}, k_{4} \in(0,1)$ such that

$$
\mid F(s, \zeta, z, u, x)-F\left(s, \zeta, \hat{z}, \hat{u}, \hat{x}\left|\leq k_{1}\right| z-\hat{z}\left|+k_{2}\right| u-\hat{u}\left|+k_{3}\right| x-\hat{u} \mid ;\right.
$$

(3) There exists $\tilde{r}>0$ such that resulting bounded fulfills

$$
\begin{aligned}
& \sup \left\{|G(s, \zeta):(s, \zeta) \in I|+\left|F\left(s, \zeta, z_{1}, z_{2}, z_{3}\right)\right|:(s, \zeta) \in I, z_{1} \in[-\tilde{r}, \tilde{r}]\right. \\
& \left.\quad z_{2} \in\left[-c d M_{1}, c d M_{1}\right], z_{3} \in\left[-c d M_{2}, c d M_{2}\right]\right\} \leq r,
\end{aligned}
$$

here

$$
\begin{aligned}
& M_{1}=\sup \left\{|g(s, \zeta, \xi, \eta, z)|: \text { for all }(s, \zeta, \xi, \eta) \in I_{2} \text { and } z \in[-\tilde{r}, \tilde{r}]\right\} \\
& M_{2}=\sup \left\{|h(s, \zeta, \xi, \eta, z)|: \text { for all }(s, \zeta, \xi, \eta) \in I_{2} \text { and } z \in[-\tilde{r}, \tilde{r}]\right\}
\end{aligned}
$$

Then

$$
\begin{aligned}
z(s, \zeta)= & G(s, \zeta)+F\left(s, \zeta, z(s, \zeta), \int_{0}^{s} \int_{0}^{\zeta} g(s, \zeta, \xi, \eta, z(\xi, \eta)) d \eta d \xi\right. \\
& \left.\int_{0}^{c} \int_{0}^{d} h(s, \zeta, \xi, \eta, z(\xi, \eta)) d \eta d \xi\right)
\end{aligned}
$$

has at least one solution in $X$.

Proof The proof is linked to the beginning Theorem 3.1 and the details that follow.

\section{Corollary 3.3 Let}

$\left(S_{1}\right) F \in C(I \times \mathbb{R} \times \mathbb{R}, \mathbb{R}), f \in C\left(I_{1}, \mathbb{R}\right), g \in C\left(I_{2} \times \mathbb{R}, \mathbb{R}\right), h \in C\left(I_{2} \times \mathbb{R}, \mathbb{R}\right) ;$

$\left(S_{2}\right)$ There exist nonnegative constants $\mu$ and $v$ such that

$$
|f(s, \zeta, 0)| \leq \mu ; \quad|F(s, \zeta, 0,0)| \leq v
$$

$\left(S_{3}\right)$ There exist nonnegative constants $k_{1}, k_{2}, k_{3} \in(0,1)$ such that

$$
\begin{aligned}
& \mid f(s, \zeta, z)-f\left(s, \zeta, \hat{z}\left|\leq k_{1}\right| z-\hat{z} \mid\right. \\
& \mid F(s, \zeta, z, u)-F\left(s, \zeta, \hat{z}, \hat{u}\left|\leq k_{2}\right| z-\hat{z}\left|+k_{3}\right| u-\hat{u} \mid\right.
\end{aligned}
$$


$\left(S_{4}\right)$ There exist nonnegative constants $c_{1}, c_{2}, d_{1}$, and $d_{2}$ such that

$$
|g(s, \zeta, \xi, \eta, z)| \leq c_{1}+c_{2}|z|, \quad|h(s, \zeta, \xi, \eta, z)| \leq d_{1}+d_{2}|z|
$$

$\left(S_{5}\right) k_{1}+k_{2} c d c_{2}+k_{3} c d d_{2}<1$.

Then the equation

$$
\begin{aligned}
z(s, \zeta)= & f(s, \zeta, z(s, \zeta))+F\left(s, \zeta, \int_{0}^{s} \int_{0}^{\zeta} g(s, \zeta, \xi, \eta, z(\xi, \eta)) d \eta d \xi\right. \\
& \left.\int_{0}^{c} \int_{0}^{d} h(s, \zeta, \xi, \eta, z(\xi, \eta)) d \eta d \xi\right)
\end{aligned}
$$

has at least one solution in $X$.

Proof Let $\tilde{r}=\frac{N_{2}}{1-N_{1}}$, where $N_{1}=k_{1}+k_{2} c d c_{2}+k_{3} c d d_{2}, N_{2}=\mu+k_{2} c d c_{1}+k_{3} c d d_{1}+v$, and

$$
G(s, \zeta)=0, \quad F(s, \zeta, z, u, x)=z+F(s, \zeta, u, x)
$$

where

$$
\begin{aligned}
& z=f(s, \zeta, z(s, \zeta)), \quad u=\int_{0}^{s} \int_{0}^{\zeta} g(s, \zeta, \xi, \eta, z(\xi, \eta)) d \eta d \xi \\
& x=\int_{0}^{c} \int_{0}^{d} h(s, \zeta, \xi, \eta, z(\xi, \eta)) d \eta d \xi
\end{aligned}
$$

(2) is conducted by $\left(S_{2}\right)$. Now, we show that $\left(S_{3}\right)$ is also fulfilled, we have

$$
\begin{aligned}
|z(s, \zeta)|= & \mid f(s, \zeta, z(s, \zeta))+F\left(s, \zeta, \int_{0}^{s} \int_{0}^{\zeta} g(s, \zeta, \xi, \eta, z(\xi, \eta)) d \eta d \xi\right. \\
& \left.\int_{0}^{c} \int_{0}^{d} h(s, \zeta, \xi, \eta, z(\xi, \eta)) d \eta d \xi\right) \mid, \\
\leq & |f(s, \zeta, z(r, \zeta))-f(s, \zeta, 0)|+|f(s, \zeta, 0)| \\
& +k_{2}\left|\int_{0}^{s} \int_{0}^{\zeta} g(s, \zeta, \xi, \eta, z(\xi, \eta)) d \eta d \xi\right| \\
& +k_{3}\left|\int_{0}^{c} \int_{0}^{d} h(s, \zeta, \xi, \eta, z(\xi, \eta)) d \eta d \xi\right|+|F(s, \zeta, 0,0)|, \\
\leq & k_{1}\|z\|+\mu+k_{2} c d\left(c_{1}+c_{2}\|z\|\right)+k_{3} c d\left(d_{1}+d_{2}\|z\|\right)+v, \\
\leq & \left(k_{1}+k_{2} c d c_{2}+k_{3} c d d_{2}\right)\|z\|+\mu+k_{2} c d c_{1}+k_{3} c d d_{1}+v
\end{aligned}
$$

for all $(s, \zeta) \in I$; consequently,

$$
\sup |F(s, \zeta, z, u, x)| \leq N_{1} r+N_{2}=N_{1} \frac{N_{2}}{1-N_{1}}+N_{2}=\tilde{r}
$$

Corollary $3.4([9])$ Let

$\left(E_{1}\right) F \in C\left(I_{1} \times \mathbb{R}, \mathbb{R}\right), g \in C\left(I_{2} \times \mathbb{R}, \mathbb{R}\right)$; 
$\left(E_{2}\right)$ There exist nonnegative constants $m_{1}$ and $m_{2}$ such that $|A(s, \zeta)| \leq m_{1} ;|F(s, \zeta, 0,0)| \leq$ $m_{2}$

$\left(E_{3}\right)$ There exist nonnegative constants $k_{1}, k_{2} \in(0,1)$ such that

$$
\mid F(s, \zeta, z, u)-F\left(s, \zeta, \hat{z}, \hat{u}\left|\leq k_{1}\right| z-\hat{z}\left|+k_{2}\right| u-\hat{u} \mid\right.
$$

$\left(E_{4}\right)$ There exist nonnegative constants $h_{1}$ and $h_{2}$ such that $|g(s, \zeta, \xi, \eta, z)| \leq h_{1}+h_{2}|z|$;

$\left(E_{5}\right) k_{1}+k_{2} c d h_{2}<1$.

Then the equation

$$
z(s, \zeta)=A(s, \zeta)+F\left(s, \zeta, z(s, \zeta), \int_{0}^{r} \int_{0}^{\zeta} g(s, \zeta, \xi, \eta, z(\xi, \eta)) d \eta d \xi\right)
$$

has at least one solution in $X$.

Proof Let $\tilde{r}=\frac{F_{2}}{1-F_{1}}$, where $F_{1}=k_{1}+k_{2} c d h_{2}, F_{2}=k_{2} c d h_{1}+m_{2}+m_{1}$, and

$$
F(s, \zeta, z, u, x)=F(s, \zeta, z, u)
$$

where

$$
z=z(s, \zeta), \quad u=\int_{0}^{s} \int_{0}^{t} g(s, \zeta, \xi, \eta, z(\xi, \eta)) d \eta d \xi
$$

$\left(T_{2}\right)$ is handled by $\left(E_{2}\right)$. Now, we show that $\left(E_{3}\right)$ is also fulfilled. We have

$$
\begin{aligned}
|z(s, \zeta)|= & \left|A(s, \zeta)+F\left(s, \zeta, z(s, \zeta), \int_{0}^{s} \int_{0}^{\zeta} g(s, \zeta, \xi, \eta, z(\xi, \eta)) d \eta d \xi\right)\right|, \\
\leq & \left|F\left(s, \zeta, z(s, \zeta), \int_{0}^{s} \int_{0}^{\zeta} g(s, \zeta, \xi, \eta, z(\xi, \eta)) d \eta d \xi\right)-F(s, \zeta, 0,0)\right| \\
& +|F(s, \zeta, 0,0)|+|A(s, \zeta)|, \\
\leq & k_{1}|z(s, \zeta)|+k_{2}\left|\int_{0}^{s} \int_{0}^{\zeta} g(s, \zeta, \xi, \eta, z(\xi, \eta)) d \eta d \xi\right| \\
& +|F(s, \zeta, 0,0)|+|A(s, \zeta)|, \\
\leq & k_{1}\|z\|+k_{2} c d\left(h_{1}+h_{2}|z|\right)+m_{2}+m_{1}, \\
\leq & \left(k_{1}+k_{2} c d h_{2}\right)\|z\|+k_{2} c d h_{1}+m_{2}+m_{1}
\end{aligned}
$$

for all $(s, \zeta) \in I$; consequently,

$$
\sup |F(s, \zeta, z, u, x)| \leq F_{1} \tilde{r}+F_{2}=F_{1} \frac{F_{2}}{1-F_{1}}+F_{2}=\tilde{r} .
$$

\section{Applications}

Example 4.1

$$
z(s, \zeta)=g(s, \zeta)+\int_{0}^{s} \int_{0}^{\zeta} P(s, \zeta, \xi, \eta) Q(\xi, \eta, z(\xi, \eta)) d \eta d \xi
$$


for $v=g(s, \zeta)$ and $h(s, \zeta, \xi, \eta, z(\xi, \eta))=P(s, \zeta, \xi, \eta) Q(\xi, \eta, z(\xi, \eta))$, which may be regarded as a two dimensional generalization of the famous Hammerstein type FIE (see [28])

$$
z(s, \zeta)=g(s, \zeta)+\int_{0}^{1} \int_{0}^{1} h(s, \zeta, \xi, \eta, z(\xi, \eta)) d \eta d \xi
$$

which is the famous two dimensional Fredholm FIE examined (e.g. [2]).

Example 4.2 Consider the following two dimensional-FIE:

$$
\begin{aligned}
z(s, \zeta)= & \frac{s^{2}}{2\left(1+s^{2} \zeta^{2}\right)} e^{-s^{2} \zeta}+\frac{1}{2}\left(\frac{1+s \zeta^{2}}{3+4 s^{2} \zeta^{2}}\right) \cos z(s, \zeta)+\frac{1}{2} \int_{0}^{s} \int_{0}^{\zeta} \xi \eta^{2} \cos z(\xi, \eta) d \eta d \xi \\
& +\frac{1}{2} \int_{0}^{1} \int_{0}^{1} \arctan \left(\frac{|z(\xi, \eta)|}{1+|z(\xi, \eta)|}\right) d \eta d \xi
\end{aligned}
$$

for $(s, \zeta) \in I=[0,1] \times[0,1]$. Here, we put

$$
\begin{aligned}
& F(s, \zeta, z, u, \eta)=\frac{1}{2} z_{+} \frac{1}{2} u+\frac{1}{2} \eta, \\
& f(s, \zeta, z)=\frac{1+s \zeta^{2}}{3+4 s^{2} \zeta^{2}} \cos z(s, \zeta), \\
& g(s, \zeta, \xi, \eta, z)=\xi \eta^{2} \cos z(\xi, \eta), \\
& h(s, \zeta, \xi, \eta, z)=\arctan \left(\frac{|z(\xi, \eta)|}{1+|z(\xi, \eta)|}\right) .
\end{aligned}
$$

It can clearly be noticed that $F, f, g, h$ are continuous functions on the respective domain and

$$
\begin{aligned}
& |F(s, \zeta, z, u, x)-F(s, \zeta, \hat{z}, \hat{u}, \hat{x})| \leq \frac{1}{2}|z-\hat{z}|+\frac{1}{2}|u-\hat{u}|+\frac{1}{2}|x-\hat{x}|, \\
& \mid f(s, \zeta, z)-f\left(s, \zeta, \hat{z}\left|\leq \frac{1}{3}\right| z-\hat{z} \mid .\right.
\end{aligned}
$$

Here, $k_{1}=k_{2}=k_{3}=k_{4}=\frac{1}{2}$. It is seen that these functions satisfy (1) and (2). Now, we check that (3) also holds. Take $r=3$, then we get $M_{1}=M_{2} \leq 1$ and

$$
\begin{aligned}
& \sup \{|G(s, \zeta)+F(s, \zeta, z, u, \eta)|: s, \zeta \in[0,1], z \in[-3,3], u, \eta \in[-1,1]\} \\
& \quad \leq \sup \mid\left(\frac{s^{2}}{2\left(1+s^{2} \zeta^{2}\right)} e^{-s^{2} \zeta}+\frac{1+s \zeta^{2}}{2\left(3+2 s^{2} \zeta^{2}\right)} \cos z(s, \zeta)+\frac{1}{2} \int_{0}^{s} \int_{0}^{\zeta} \xi \eta^{2} \cos z(\xi, \eta) d \eta d \xi\right. \\
& \left.\quad+\frac{1}{2} \int_{0}^{1} \int_{0}^{1} \arctan \left(\frac{|z(\xi, \eta)|}{1+|z(\xi, \eta)|}\right) d \eta d \xi\right) \mid \\
& \leq 3
\end{aligned}
$$

All assumptions (1)-(3) are satisfied. Hence, by Theorem 3.1, equation (7) has at least one solution in $C(I)$. 


\section{Conclusion}

By unifying and enlarging the earlier results of $[9,11,18,35]$ and using Petryshyn's fixed point Theorem 3.1, in the third section, we obtained a new method to prove the existence of solutions for some functional integral equations. The merit of Theorem 3.1 among the others (Darbo's and Schauder's fixed point theorems) lies in that in applying the theorem, one does not need to confirm that the involved operator maps a closed convex subset onto itself. For future work, the interested researchers can obtain the existence of solution of equation (1) in different Banach function spaces e.g. Sobolev space, Hölder space, etc.

\section{Acknowledgements}

The authors extend their appreciation to the Deanship of Scientific Research at King Khalid University for funding this work through Research Group Program under grant number RGP. 2/5/42

Availability of data and materials

Not applicable.

\section{Competing interests}

The authors declare that they have no competing interests.

\section{Authors' contributions}

All the authors contributed equally and they read and approved the final manuscript for publication.

\section{Author details}

'Department of Applied Sciences and Engineering, IIT Roorkee, Roorkee 247667, India. ${ }^{2}$ Department of Mathematics, Meerut College Meerut, Meerut 250001, India. ${ }^{3}$ Department of Mathematics, College of Arts and Sciences, Prince Sattam bin Abdulaziz University, Wadi Aldawaser, Saudi Arabia. ${ }^{4}$ Department of Mathematics, College of Science, King Khalid University, P.O. Box 9004, Abha 61413, Saudi Arabia. ${ }^{5}$ Department of Engineering Mathematics and Physics, Faculty of Engineering, Al-Azhar University, Cairo, Egypt. ${ }^{6}$ Department of Mathematics, Faculty of Science, Al-Azhar University, 71524, Assiut, Egypt.

\section{Publisher's Note}

Springer Nature remains neutral with regard to jurisdictional claims in published maps and institutional affiliations.

Received: 25 March 2021 Accepted: 12 July 2021 Published online: 09 August 2021

\section{References}

1. Amar, A.B., Jeribi, A., Mnif, M.: Some fixed point theorems and application to biological model. Numer. Funct. Anal. Optim. 29(1-2), 1-23 (2008)

2. Babaaghaie, A., Maleknejad, K.: A new approach for numerical solution of two-dimensional nonlinear Fredholm integral equations in the most general kind of kernel, based on Bernstein polynomials and its convergence analysis. J. Comput. Appl. Math. 344, 482-494 (2018)

3. Banaś, J., Goebel, K.: Measures of Noncompactness in Banach Spaces. Dekker, New York (1980)

4. Banaś, J., Lecko, M.: Fixed points of the product of operators in Banach algebra. Panam. Math. J. 12, 101-109 (2002)

5. Banaś, J., Rzepka, B.: On existence and asymptotic stability of solutions of a nonlinear integral equation. J. Math. Anal. Appl. 284, 165-173 (2003)

6. Banaś, J., Sadarangani, K.: Solutions of some functional integral equations in Banach algebra. Math. Comput. Model. 38, 245-250 (2003)

7. Baradol, P., Gopal, D., Radenovic, S.: Computational fixed points in graphical rectangular metric spaces and an application to integral equation. J. Comput. Appl. Math. 375, 112805 (2020)

8. Das, A., Hazarika, B., Arab, R., Mursaleen, M.: Applications of a fixed point theorem for the existence of solutions of nonlinear functional integral equations in two variables. Rend. Circ. Mat. Palermo 2(68), 139-152 (2019)

9. Das, A., Hazarika, B., Kumam, P.: Some new generalization of Darbo's fixed point theorem and its application on integral equations. Mathematics 7(3), 214 (2019)

10. Deep Deepmala, A., Ezzati, R.: Application of Petryshyn's fixed point theorem to solvability for functional integral equations. Appl. Math. Comput. 395, 125878 (2021)

11. Deep Deepmala, A., Rabbani, M.: A numerical method for solvability of some non-linear functional integral equations. Appl. Math. Comput. 402, 125637 (2021)

12. Deep Deepmala, A., Roshan, J.R., Nisar, K.S., Abdeljawad, T.: An extension of Darbo's fixed point theorem for a class of system of nonlinear integral equations. Adv. Differ. Equ. 2020, 483 (2020)

13. Deepmala, Pathak, H.K.: A study on some problems on existence of solutions for some nonlinear functional-integral equations. Acta Math. Sci. 33(5), 1305-1313 (2013)

14. Dhage, B.C.: On $\alpha$-condensing mappings in Banach algebras. Math. Stud. 63, 146-152 (1994)

15. Fabiano, N., Nikolic, N., Shanmugam, T., Radenovic, S., Citakovic, N.: Tenth order boundary value problem solution existence by fixed point theorem. J. Inequal. Appl. 2020, 166 (2020)

16. Goldenstein, L.S., Markus, A.S.: On the measure of non-compactness of bounded sets and of linear operators. In: Studies in Algebra and Math. Anal., pp. 45-54. Izdat. Karta Moldovenjaske, Kishinev (1965) (Russian) 
17. Hazarika, B., Arab, R., Nashine, H.K.: Applications of measure of noncompactness and modified simulation function for solvability of nonlinear functional integral equations. Filomat 33(17), 5427-5439 (2019)

18. Hazarika, B., Srivastava, H.M., Arab, R., Rabbani, M.: Application of simulation function and measure of noncompactness for solvability of nonlinear functional integral equations and introduction to an iteration algorithm to find solution. Appl. Math. Comput. 360, 131-146 (2019)

19. Hussain, S., Latif, M.A., Alomari, M.: Generalized double-integral Ostrowski type inequalities on time scales. Appl. Math. Lett. 24(8), 1461-1467 (2011)

20. Jeribi, A.: A nonlinear problem arising in the theory of growing cell populations. Nonlinear Anal., Real World Appl. 3(1), 85-105 (2002)

21. Kazemi, M., Ezzati, R.: Existence of solutions for some nonlinear two dimensional Volterra integral equations via measures of noncompactness. Appl. Math. Comput. 275, 165-171 (2016)

22. Kazemi, M., Ezzati, R.: Existence of solutions for some nonlinear Volterra integral equations via Petryshyn's fixed point theorem. Int. J. Anal. Appl. 9, 1-12 (2018)

23. Kuratowski, K.: Sur les espaces completes. Fundam. Math. 15, 301-335 (1934)

24. Maleknejad, K., Mollapourasl, R., Nouri, K.: Study on existence of solutions for some nonlinear functional integral equations. Nonlinear Anal. 69, 2582-2588 (2008)

25. Maleknejad, K., Nouri, K., Mollapourasl, R.: Existence of solutions for some nonlinear integral equations. Commun. Nonlinear Sci. Numer. Simul. 14, 2559-2564 (2009)

26. Manam, S.R.: Multiple integral equations arising in the theory of water waves. Appl. Math. Lett. 24(8), 1369-1373 (2011)

27. Nussbaum, R.D.: The fixed point index and fixed point theorem for $k$ set contractions, Thesis (Ph.D), - The University of Chicago, Proquest LLC, Ann Arbor, (1969)

28. Pachpatte, B.G.: Multidimensional Integral Equations and Inequalities. Atlantis Press, Paris (2011)

29. Petryshyn, W.V.: Structure of the fixed points sets of $k$-set-contractions. Arch. Ration. Mech. Anal. 40, 312-328 (1970-1971)

30. Rabbani, M., Arab, R., Hazarika, B.: Solvability of nonlinear quadratic integral equation by using simulation type condensing operator and measure of noncompactness. Appl. Math. Comput. 349, 102-117 (2019)

31. Rabbani, M., Deep Deepmala, A.: On some generalized non-linear functional integral equations of two variables via measures of noncompactness and numerical method to solve it. Math. Sci., 1-8 (2021)

32. Roshan, J.R.: Existence of solutions for a class of system of functional integral equation via measure of noncompactness. J. Comput. Appl. Math. 313, 129-141 (2017)

33. Sarwar, M., Bahadur Zada, M., Radenovic, S.: Rational type inequality with applications to Volterra-Hammerstein nonlinear integral equations. Int. J. Nonlinear Sci. Numer. Simul. 15(5), 465-473 (2020). https://doi.org/10.1515/ijnsns-2018-0367

34. Sen, M., Saha, D., Agarwal, R.P.: A Darbo's fixed point theory approach towards the existence of a functional integral equation in a Banach algebra. Appl. Math. Comput. 358, 111-118 (2019)

35. Srivastava, H.M., Das, A., Hazarika, B., Kutbi, M.A., Mohiuddine, S.A.: Existence of solution for nonlinear functional integral equations of two variables in Banach algebra. Symmetry 11(5), 674 (2019)

\section{Submit your manuscript to a SpringerOpen ${ }^{\circ}$ journal and benefit from:}

- Convenient online submission

- Rigorous peer review

- Open access: articles freely available online

- High visibility within the field

- Retaining the copyright to your article

Submit your next manuscript at $\gg$ springeropen.com 